\title{
Molecular similarities and differences from human pulmonary fibrosis and corresponding mouse model: MALDI imaging mass spectrometry in comparative medicine
}

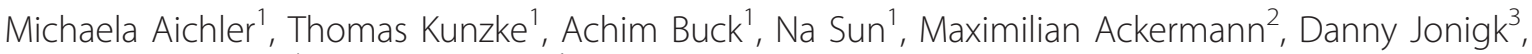
Andreas Gaumann ${ }^{4}$ and Axel Walch ${ }^{1}$

Animal models can reproduce some model-specific aspects of human diseases, but some animal models translate poorly or fail to translate to the corresponding human disease. Here, we develop a strategy to systematically compare human and mouse tissues, and conduct a proof-of-concept experiment to identify molecular similarities and differences using patients with idiopathic pulmonary fibrosis and a bleomycin-induced fibrosis mouse model. Our novel approach employs highthroughput tissue microarrays (TMAs) of humans and mice, high-resolution matrix-assisted laser desorption/ionizationFourier transform-ion cyclotron resonance-mass spectrometry imaging (MALDI-FT-ICR-MSI) to spatially resolve mass spectra at the level of specific metabolites, and hierarchical clustering and pathway enrichment analysis to identify functionally similar/different molecular patterns and pathways in pathological lesions of humans and mice. We identified a large number of common molecules $(n=1366)$ and fewer exclusive molecules in humans $(n=83)$ and mice $(n=54)$. Among the common molecules, the 'ascorbate and aldarate metabolism' pathway had the highest similarity in human and mouse lesions. This proof-of-concept study demonstrates that our novel strategy employing a reliable and easy-toperform experimental design accurately identifies pathways and factors that can be directly compared between animal models and human diseases.

Laboratory Investigation (2018) 98, 141-149; doi:10.1038/labinvest.2017.110; published online 16 October 2017

The scientific community has questioned the translation of experimental results from induced or genetically modified animal models to human diseases, which casts potential doubt on the translation of results from bench-to-bedside. In the field of medical research, animal models are considered as valuable research tools that provide insights into the complex world of human diseases. However, a model is only a simple representation of a complex system. ${ }^{1}$ The ease with which animal models allow advanced research entails a risk of ignoring the possibility that the results from those studies may only provide limited translational potential to humans. ${ }^{2}$ An animal model may not reproduce a human disease with all its complexities, ${ }^{1}$ although it may reproduce model-specific aspects of a disease. ${ }^{1}$ Thus, it is of utmost importance to define the aspects of a human disease that are accurately represented by a specific animal model. This knowledge is crucial to ensure that an animal model is suitable for the purposes of an experimental investigation.

Direct molecular comparisons between human diseases and animal models are limited and demonstrate both good translation and impossible translation. One of the most impressive studies that reported a failure in the translation from an animal model to humans involved a mouse model of genomic response to inflammation, which poorly mimicked human inflammatory disease. ${ }^{3}$ Conversely, several studies in genetically engineered mouse models have shown promising initial results for clinical challenges in diagnosis, treatment, and research. ${ }^{4}$ Histopathological examination plays a key role in comparative studies to identify similarities between human diseases and their corresponding animal models. A

${ }^{1}$ Research Unit Analytical Pathology, Helmholtz Zentrum München, Oberschleißheim, Germany; ${ }^{2}$ Institute of Functional and Clinical Anatomy, University Medical Center of the Johannes Gutenberg University Mainz, Mainz, Germany; ${ }^{3}$ Institute of Pathology, Medizinische Hochschule Hannover, Hannover, Germany and ${ }^{4}$ Institute of Pathology Kaufbeuren-Ravensburg, Kaufbeuren, Germany

Correspondence: Professor A Walch, MD, Research Unit Analytical Pathology, Helmholtz Zentrum München, Ingolstädter Landstraße 1, Oberschleißheim-Neuherberg 85764, Germany. Email: axel.walch@helmholtz-muenchen.de

Received 22 May 2017; revised 17 August 2017; accepted 22 August 2017 
breakthrough occurred in a comparative study of a mouse model for pancreatic ductal adenocarcinoma, in which mice have similar genetic alterations as in the human disease and develop pancreatic cancer that was histologically comparable with that in humans. ${ }^{4-7}$ Although histopathology is useful for these comparative studies, molecular analyses in these studies are limited.

Many advanced technologies are currently used for tissuebased research. Mass spectrometry has unique advantages among these technologies, including high sensitivity, wide range of molecules, molecular specificity, and the flexibility to identify numerous and varied analytes on a single platform. Most mass spectrometric techniques require liquid samples derived from homogenized tissues. However, tissue samples are not homogeneous and consist of multiple different cell types. Thus, small pathological lesions only constitute a small percentage of the overall tissue sample, and high-throughput techniques do not employ procedures that enable specific investigation of these small tissue subsets. One way to overcome this limitation is to perform tissue microdissection and concentrate the lesions of interest ${ }^{8,9}$ this is very labor intensive and requires large samples, which are not often available. Matrix-assisted laser desorption/ionization mass spectrometry imaging (MALDI-MSI) is a powerful technique that combines mass spectrometry molecular analysis with histology, which enables spatially resolved and label-free detection of hundreds to thousands of molecules within a single tissue section. ${ }^{10,11}$ After performing the initial MALDIMSI measurements, computational microdissection of molecular signatures in the lesions of interest generates spatially resolved metabolic maps that can be analyzed in greater detail. ${ }^{10,11}$

In the present study, a specialized statistical approach of the MALDI imaging data plays an important role. To elucidate molecular similarities and differences between mouse and human tissues, we perform a hierarchical clustering analysis, which is a well-established statistical method within the context of tumor heterogeneity. ${ }^{12}$ Thus, the most important feature of MALDI imaging is analysis of the spatially resolved mass spectrometric data. We segmented the lesions of interest and then performed hierarchical clustering analysis to generate molecular patterns of functional similarity or difference. ${ }^{13}$ We used this analytical method to identify functionally similar and functionally differences between human patients and model mice, and then conducted deeper molecular analyses.

We recently published a new protocol for molecular imaging using high-resolution matrix-assisted laser desorption/ionization-Fourier transform-ion cyclotron resonancemass spectrometry imaging (MALDI-FT-ICR-MSI). ${ }^{14,15}$ This analytical approach enables the molecular in situ imaging of cellular metabolites in formalin-fixed paraffin-embedded (FFPE) tissue samples for the first time. The protocol generates multiplex molecular data with unprecedented detail. It can be used to analyze archived tissue material, which enables direct molecular comparison of archived patient tissues with corresponding model animal tissues. In the present study, we constructed tissue microarrays (TMAs) using archived FFPE tissues from patients with idiopathic pulmonary fibrosis and FFPE tissues from the corresponding bleomycin-induced mouse model. TMAs enable large numbers of individual patients and individual mice to be screened in the same experiment with one MALDI-FT-ICR-MSI measurement. This ensures that all specimens are analyzed using the very same experimental conditions, which is a prerequisite for our experimental approach. This strategy enables direct comparisons of the results for each individual with the results for all other individuals within the same experiment. The possibility of combining large numbers of tissue samples from patients or animals into one FFPE block also enables high-throughput sample screening to define statistically and biologically reliable molecular patterns.

The principle goal of this proof-of-concept study was to create a novel approach for identifying factors in human diseases and their corresponding animal models that could be directly compared with each other using a reliable and easyto-perform experimental protocol. We present a protocol that unites classical histological techniques with highly sophisticated tissue-based molecular analyses using high-resolution MALDI imaging and subsequent statistical analyses. This enables the investigation of a broad range of disease-related aspects that have been translated from humans into the mouse model or vice versa (Figure 1a). Our experimental setup enables comprehensive comparative molecular analyses within one experiment for the first time, which avoids interexperimental variations and saves cost and time. We consider this approach as a novel solution to validate a given animal model for specific purposes. The designed experimental pipeline can be easily standardized and displays excellent reproducibility. ${ }^{15}$ Our approach will help researchers obtain more reliable results from animal experiments.

In this proof-of-concept study, we validated metabolomic characteristics of bleomycin-induced pulmonary fibrosis in mice by direct comparison with metabolomic characteristics of pulmonary fibrosis in patients to identify molecular similarities and differences between the animal model and humans.

\section{MATERIALS AND METHODS Tissue Preparation}

Primary surgical resection specimens were obtained from patients diagnosed with lung fibrosis $(n=10)$. Samples were collected at the Institute of Pathology, Hannover Medical School, Germany. All experiments were performed in accordance with relevant guidelines and regulations. All tissue specimens analyzed in this study were obtained from patients who gave their written informed consent and the study was approved by the Ethics Committee of the Hannover Medical School (ethical votes \#1691-2013 and \#3381-2016). 


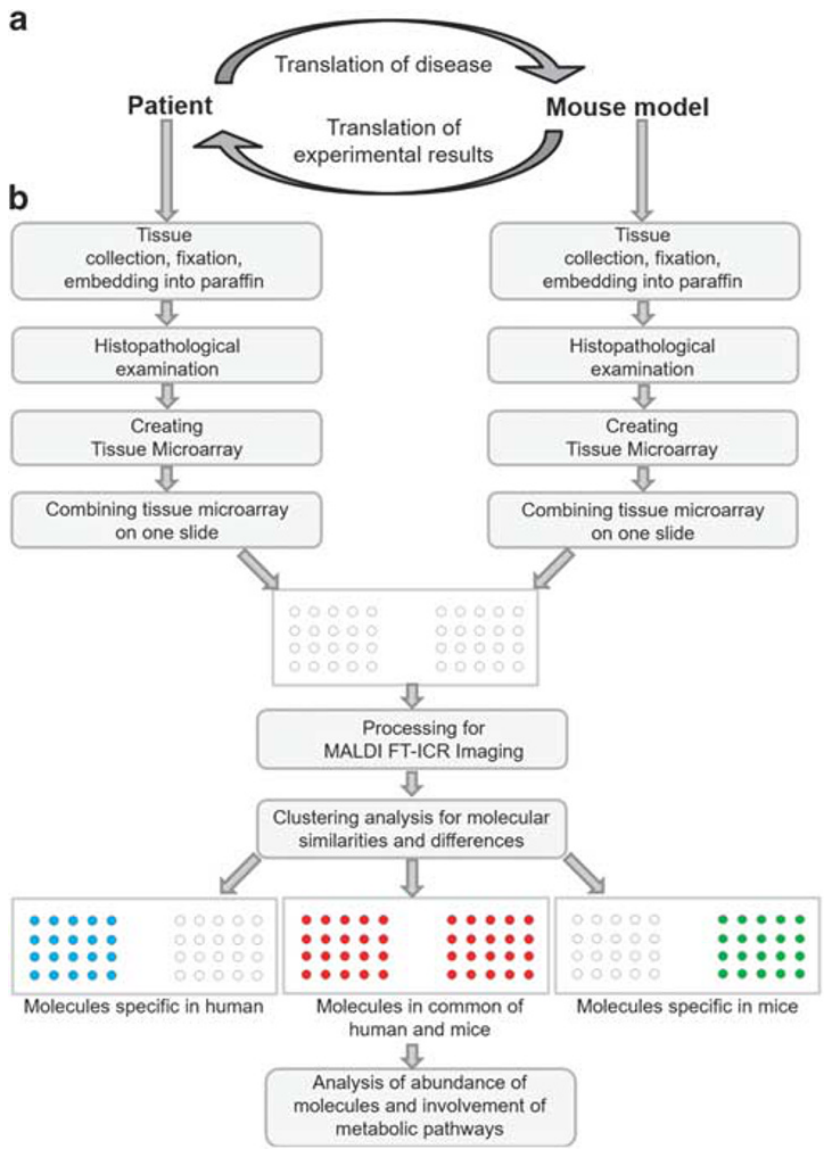

Figure 1 Schematic of the experimental protocol. (a) This novel experimental protocol enables direct comparison of molecular aspects of human diseases with their corresponding animal models, and vice versa. (b) Principal workflow of the experimental protocol, which achieves a unique synergism of histology, mass spectrometric imaging, and sophisticated statistical analysis.

Bleomycin-induced fibrotic mice $(n=23)$ also were used. The care of the animals was consistent with guidelines of the German Federal Association for Accreditation of Laboratory Animal Care. Induction of bleomycin-induced lung fibrosis was intratracheally instilled and animal housing were carried out according standardized procedures by bleomycin (Calbiochem, Darmstadt, Germany) at a dose of $0.5 \mathrm{mg} / \mathrm{kg}$ (1.5-2 U/mg) in sterile isotonic saline (50 $\mu \mathrm{l}$ per animal). All methods were performed in accordance with the relevant guidelines and regulations.

Tissues were prepared for MALDI-FT-ICR-MSI analysis by tissue sectioning, deparaffinisation, and matrix coating. FFPE tissue samples were sectioned with a thickness of $3 \mu \mathrm{m}$, and water-bath mounted onto indium-tin oxide (ITO)-coated glass slides (Bruker Daltonik GmbH, Bremen, Germany) pretreated with 1:1 poly-L-lysine (Sigma-Aldrich, Munich, Germany) and 0.1\% Nonidet P-40 (Sigma). FFPE sections were incubated for $1 \mathrm{~h}$ at $70^{\circ} \mathrm{C}$, deparaffinized in xylene $(2 \times 8 \mathrm{~min})$, and air dried. Briefly, FFPE samples were coated in $10 \mathrm{mg} / \mathrm{ml}$ 9-aminoacridine matrix in $70 \%$ methanol using a SunCollect sprayer (Sunchrom, Friedrichsdorf, Germany) as described previously. ${ }^{14,15}$

\section{MALDI-FT-ICR-MSI}

MALDI-FT-ICR-MSI (solariX 7 T FTICR MS, Bruker) measurements were performed in the mass range of $\mathrm{m} / \mathrm{z}$ 50-1000 in negative ion mode with an estimated resolution of 35000 at $\mathrm{m} / z 400$ and a spatial resolution of $60 \mu \mathrm{m}$. Spectra were recorded on solariXcontrol software with $1 \mathrm{M}$ data points, a transient length of $0.26 \mathrm{~s}$, and an ion cooling time of $0.01 \mathrm{~s}$. Source conditions were as follows: deflector plate at $-200 \mathrm{~V}$, funnel 1 at $-150 \mathrm{~V}$, skimmer 1 at $-15 \mathrm{~V}$, and funnel rf amplitude of $150 \mathrm{Vpp}$. For each measurement position, 40 laser shots were accumulated using a Smartbeam-II Nd:YAG $(355 \mathrm{~nm})$ laser with an operating at a frequency of $500 \mathrm{~Hz}$. Mass spectrometer calibration was conducted using L-arginine in the electrospray ionization (ESI) mode. A non-tissue measurement region was included as background control. Following measurement, the matrix was removed with $70 \%$ ethanol, stained with H\&E, mounted with a coverslip, scanned with a Mirax Desk scanner (Zeiss, Göttingen, Germany) with a $\times 20$ magnification objective, and co-registered to respective MSI data using FlexImaging v. 4.0 (Bruker). FlexImaging was used to annotate tissues by defining regions of interest and to export spectral data normalized with respect to root mean square as described previously. ${ }^{14,15}$

\section{Immunohistochemistry for Validation}

Immunohistochemical staining was performed on a Discovery XT automated stainer (Ventana, Tucson, AZ, USA). As a primary antibody Anti-Chondroitin Sulfate antibody (monoclonal mouse, Nr: SAB420096, Sigma) was used. The secondary antibody was a universal secondary antibody (Roche: 05268877001 ). Slides were scanned at $\times 20$ objective magnification using a Mirax Desk digital slide scanner (Carl Zeiss MicroImaging, Munich, Germany).

\section{Bioinformatics and Statistical Analysis}

Data processing was performed with a MATLAB script using the bioinformatics and image processing toolboxes (v.7.10.0, MathWorks, Natick, MA, USA). All exported spectra underwent baseline subtraction to remove noise, resampling to lower data dimensionality, and smoothing to remove tissue and measurement artefacts. ${ }^{16}$ Peak picking was conducted using a modified version of the LIMPIC algorithm, ${ }^{17}$ using $\mathrm{m} / \mathrm{z} 0.0005$ minimal peak width, a signal-to-noise threshold of 2 , and an intensity threshold of $0.01 \%$. Peaks were picked if found in $80 \%$ of the samples in each group. Isotopes were automatically identified and excluded. To identify statistically significant differences in $\mathrm{m} / \mathrm{z}$ values, the peak lists were analyzed with the Mann-Whitney $U$-test (alpha $=0.05$ ). Then, $\mathrm{m} / \mathrm{z}$ values that also occurred in the non-tissue measurement region (acting as a background control) were excluded from these peak lists by assuming that $\mathrm{m} / z$ values 
occurring in the non-tissue region could be molecules from the slide surface.

\section{RESULTS}

\section{Direct Comparison of Fibrotic Lesions in Humans and an Induced Mouse Model}

A number of mouse models have been developed to mimic key features of pulmonary fibrosis by triggering lung injuries; however, none of the mouse models fully recreate the chronic aspect of the disease. ${ }^{18}$ Bleomycin is the most frequently used agent, which is regarded as the standard for modeling fibrosis in mice. We directly compared tissues from patients with idiopathic pulmonary fibrosis with tissues from bleomycininduced pulmonary fibrosis in mice to identify specific similarities and differences in the fibrotic lesions.

A schematic of our experimental approach is presented in Figure 1b. The standard procedure for preserving and archiving patient tissue is formalin fixation followed by embedding in paraffin (FFPE) as described previously. ${ }^{15}$ To compare human and mouse tissue samples, it is essential that all tissues are processed in the same way (Figure 1b). Thus, tissues were excised from bleomycin-treated mice, fixed in formalin, and embedded in paraffin. Another important step is to define morphological comparable regions in the two tissue samples. Thus, each tissue block was sliced into $3 \mu \mathrm{m}$ thick sections, stained with hematoxylin and eosin (H\&E), subjected to pathological examination, and comparable tissue regions were marked. This was the entry point for generating TMAs, which consisted of separate tissue cores from the marked regions of each sample that were assembled as an array to allow multiplex histological analysis of a large number of specimens at the same time. We created two TMAs, one for patient tissue and one for mouse tissue. Finally, the TMAs were sliced into $3 \mu \mathrm{m}$-thick sections. One slice of the patient TMA and one slice of the mouse TMA were combined on the same MALDI imaging glass slide. The combined TMA sections were simultaneously imaged with MALDI-FT-ICR-MSI and measured within a single run as described in our recently published protocol. ${ }^{14,15}$

\section{Distribution of Molecules in Fibrotic Lesions of Human and Mouse Tissues}

After performing MALDI-FT-ICR MSI measurements, the matrix was removed from the tissue sections, the samples were stained with $\mathrm{H} \& \mathrm{E}$, and then the samples were coregistered to the mass spectrometric data. Fibrotic areas were marked as regions of interest, and fibrosis-specific mass spectra were extracted. We identified molecules that were characteristic for humans ( $n=83$ molecules) or mice ( $n=54$ molecules; Figure 2). The majority of identified molecules were common in humans and mice $(n=1366$ molecules; Figure 2).

Next, we performed an unsupervised hierarchical clustering of the mass spectral data to spatially resolve molecular components and identify functionally similar regions in

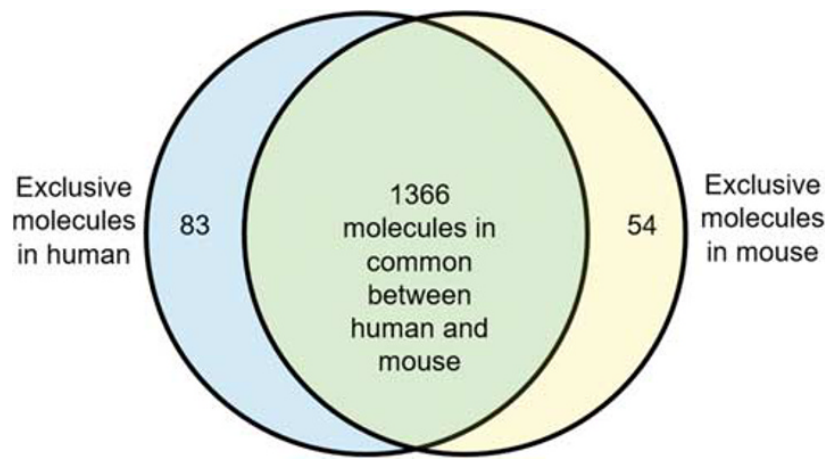

Figure 2 Numbers of specific or shared molecules in the fibrotic tissues of humans and mice. Venn diagram demonstrating the large numbers of shared molecules in common in human idopathic pulmonary fibrosis and the bleomycin-induced mouse model.

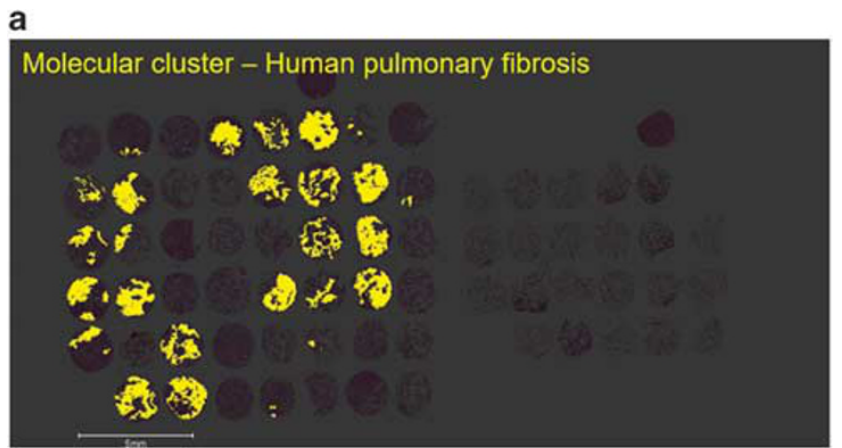

b

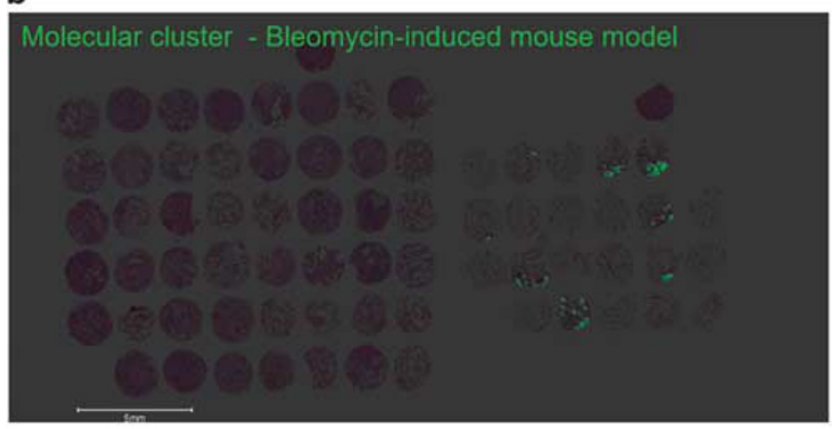

C

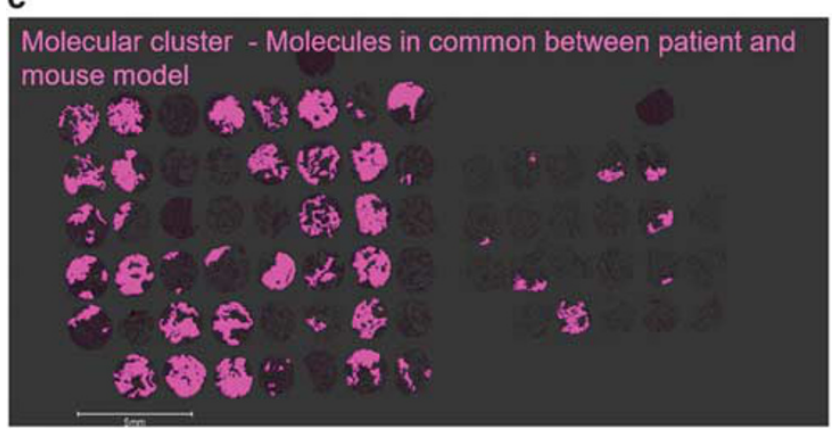

Figure 3 Molecular clustering of molecules that are specific or shared in fibrotic tissues of humans and mice. (a) Molecular characteristics that are specific for human idopathic pulmonary fibrosis. (b) Molecular characteristics that are specific for the bleomycin-induced mouse model. (c) Molecular characteristics that are shared in common in human idiopathic pulmonary fibrosis and the bleomycin-induced mouse model. 
human and bleomycin-induced mouse fibrotic tissues. We identified three distinct molecular clusters, which provided information on characteristic molecules in human tissue (Figure 3a), characteristic molecules in bleomycin-induced mouse tissue (Figure 3b), and characteristic molecules that were common in human and mouse tissues (Figure 3c). For example, the molecular distribution of a chondroitin/ dermatan fragment $(\mathrm{m} / \mathrm{z} 378.1031)$ and chondroitin/dermatan sulfate fragment was common in both human and mouse tissues (Figures $4 \mathrm{a}$ and $\mathrm{b}$ ). We validated by immunohistochemical staining on the TMA the local distribution of chondroitin sulfate of which we have also presented the MALDI imaging data. (Figures $4 \mathrm{~b}$ and 5). The MALDI-FTICR-MSI signal and the immunohistochemical staining demonstrated a high degree of overlapping localization (Figure 5).

\section{Common Molecules in Fibrotic Lesions of Human and Mouse Tissues}

To further characterize the shared molecular patterns in human and mouse tissues, we analyzed the abundance of the 1366 common molecules. The abundance of these molecules in mice was normalized with respect to the relative intensity of the molecular ion peaks in humans. Molecules that were
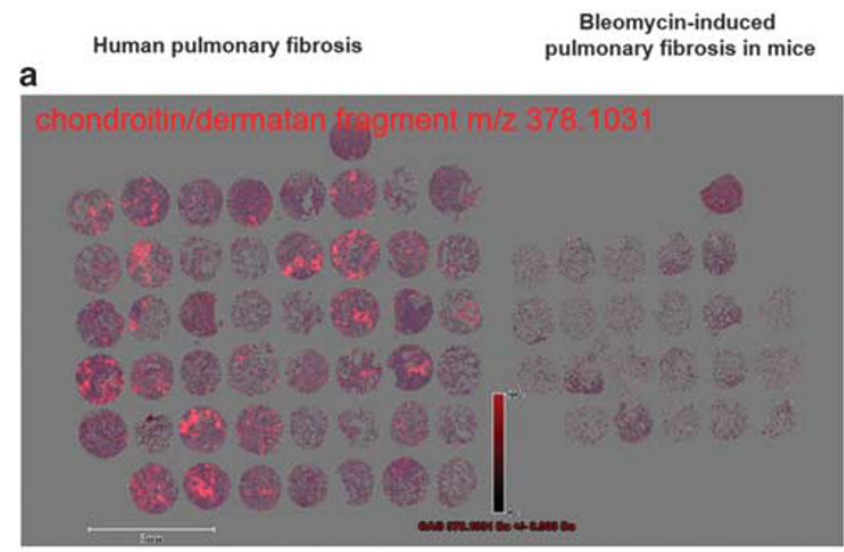

b

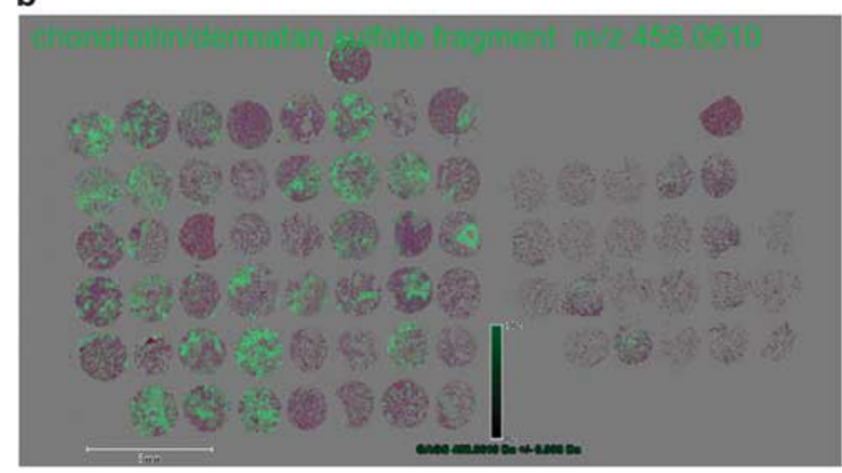

Figure 4 Abundance and spatial distribution of glycosaminoglycans. (a) Chondroitin/dermatan fragments and (b) chondroitin/dermatan sulfate fragments are abundant in fibrotic tissues of both human idiopathic pulmonary fibrosis and the related mouse model. detected in mice tissue and were $<50 \%$ of the relative intensity in humans were considered as having lower abundance, molecules in the range of $50-200 \%$ of the relative intensity in humans were considered as equally abundant, and molecules in the range of $>200 \%$ of the relative intensity in humans were considered as having higher abundance. Using this categorization we identified 900 molecules that were equally abundant in humans and mice, 282 molecules that had lower abundance in mice than in humans, and 184 molecules that had higher abundance in mice than in humans (Figures $6 a$ and $b$ ).

\section{Common Metabolic Pathways in Fibrotic Lesions of Human and Mouse Tissues}

The 900 molecules that were comparably abundant in human and mouse tissues were annotated by alignment with the Metlin and KEGG databases (https://metlin.scripps.edu/ index.php, http://www.kegg.jp). Then, these results were subjected to pathway enrichment analysis (http://www. metaboanalyst.ca) to determine which pathways in the induced mouse tissue were comparable with those in diseased human tissue. We identified six pathways that had the highest enrichment (Figure 7), including tryptophan metabolism, galactose metabolism, starch and sucrose metabolism, glyoxylate and dicarboxylate metabolism, pentose and glucuronate interconversions, and ascorbate and aldarate metabolism. The ascorbate and aldarate metabolic pathway was the most prominent pathway among these six pathways, which could provide a basis for further investigations.

\section{DISCUSSION}

Studies in which the translation of results from animal experiments to humans failed have raised serious questions about the value of animal models and the need to validate established animal models. It is clearly necessary to establish a new understanding of the limitations of animal models and the validated extrapolations that can be formalized from animal models. Thus, we developed a new experimental approach that enables direct comparisons of animal models with humans in a very easy and reliable way. The present study provides a proof-of-concept experiment (Figure 1) that validates our protocol. This study directly compares fibrotic tissues from humans and induced mice and identifies molecular similarities and differences in these two tissues. Our novel protocol can be implemented to obtain molecular information that allows researchers to determine the specific aspects that are common and that differ between human diseases and animal models. Or, vice versa, what aspects of a human disease are missing and have to be improved in the model. Our protocol enables a multiplex study of hundreds to thousands of molecules in human and animal tissues within a single experiment. We recently published a protocol for spatially resolved mass spectrometry of metabolites in FFPE tissues. ${ }^{14,15}$ The present study incorporates preserved human specimens from archival material, thereby opening an 


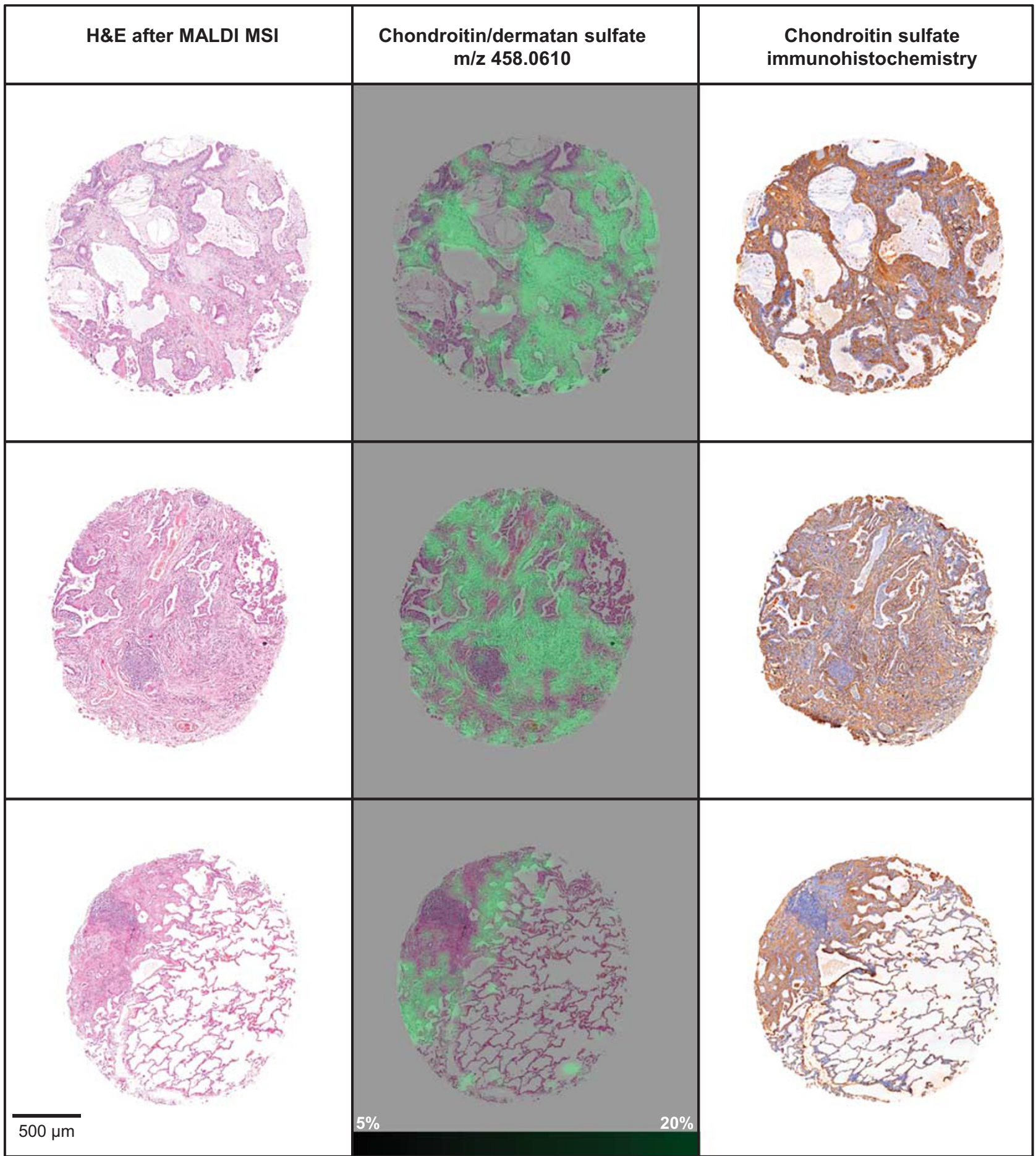

Figure 5 Immunohistochemical validation of chondroitin sulfate. The MALDI-FT-ICR-MSI signal and the immunohistochemical staining demonstrate a high degree of overlapping localization in fibrosis. (a) H\&E stained section of a lung with pulmonary fibrosis. (b) Same H\&E stained section with merged MALDI-FT-ICR signal for chondroitin/dermatan sulfate (green). (c) Immunohistochemical validation for chondroitin sulfate.

unprecedented source for comparative medical research. The use of tissue microarrays theoretically enables highthroughput analysis of archived specimens to generate statistically and biologically reliable results. The protocol ensures high comparability between all individual tissue cores because all samples are measured in the same imaging run.

This proof-of-concept study compares tissues from human patients with idiopathic pulmonary fibrosis with tissues from 
a

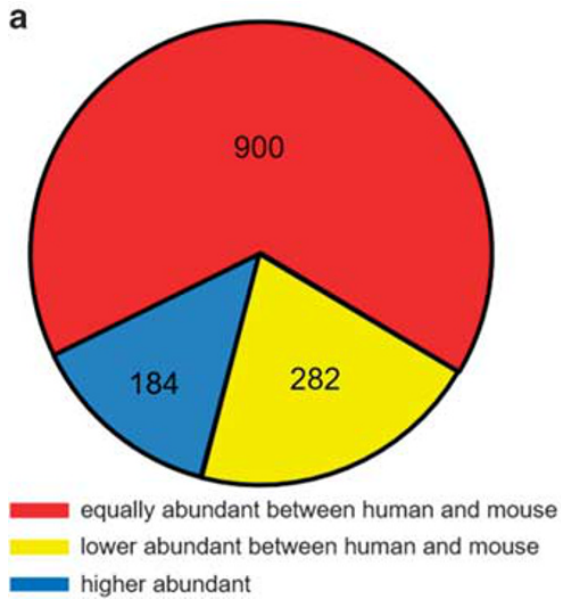

b

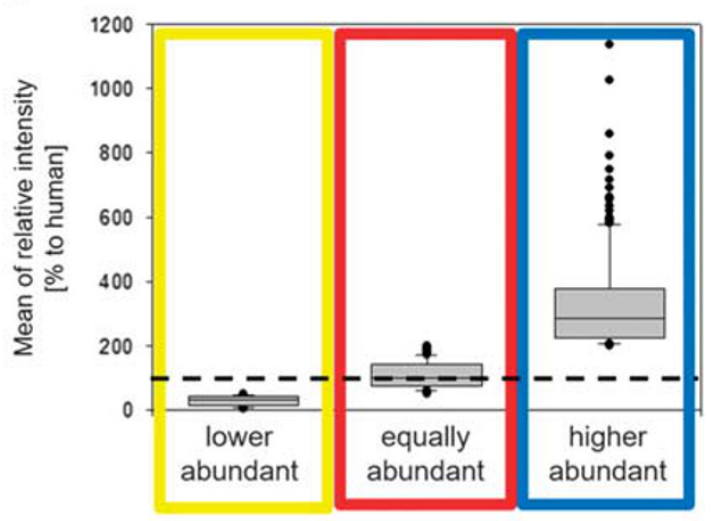

Figure 6 Abundance of molecules in fibrotic tissues of humans and mice. (a) Numbers of molecules that are comparably abundant or different for humans and/or mice. (b) Abundance of mouse-derived molecules with respect to those in patients. Dotted line represents normalization to $100 \%$ molecular abundance of molecules in humans.

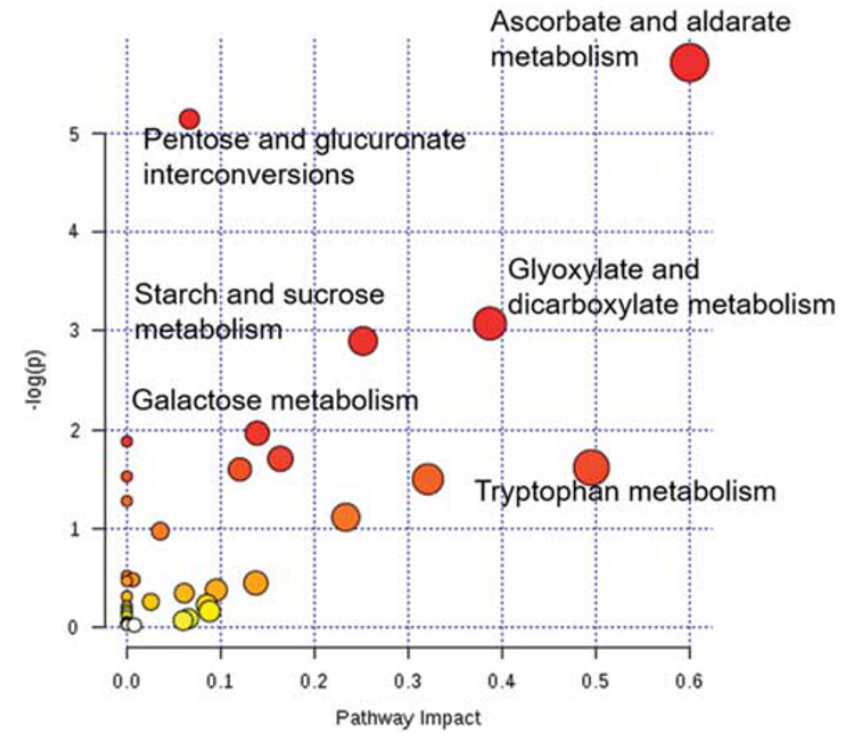

Figure 7 Enrichment analysis of metabolic pathways in fibrotic tissues of humans and mice. Estimation of common metabolic pathways that are shared in humans and mice.

bleomycin-induced mice with pulmonary fibrosis. We identified moderate numbers of molecules that were specific for either humans or mice and a large number of molecules that were commonly shared in humans and mice (Figure 2). Within the common molecules, we annotated a chondroitin/ dermatan sulfate fragment (Figure 4). Pulmonary fibrosis is characterized by increased proteoglycan deposition, which leads to loss of normal lung structure and function. ${ }^{19,20}$ Proteoglycans are key components of the lung extracellular matrix, and are characterized by the presence of one or multiple glycosaminoglycan side chains. ${ }^{21}$ Glycosaminoglycans are key players in tissue repair and fibrosis. ${ }^{22-25}$ There is an increased deposition of the chondroitin/dermatan sulfate glycosaminoglycan in pulmonary fibrosis. ${ }^{21}$ Thus, our
MALDI-FT-ICR-MSI results and analyses are consistent with previous reports. An interesting future prospect would be the analysis of glycan fragments, eg, in the blood. Based on the high abundance of glycans observed in tumor stroma regions and the apparent correlation with tumor biology, ${ }^{26}$ current findings indicate that glycan fragments could prove to be useful biomarkers. Changing of glycosylation in cystic fluids is already showing a potential for ovarian tumor staging and classification. ${ }^{27}$

Most of the shared molecules were comparably abundant in the two tissues (Figures 6a and b), which suggests a highly functional molecular overlap between fibrotic disease in humans and the induced fibrotic mouse model. Molecules that were comparably abundant in humans and mice were annotated and subsequently analyzed for pathway enrichment. The results identified common metabolic pathways in fibrotic tissues of humans and mice. We found that the ascorbate and aldarate metabolism pathway was most enriched in these tissues (Figure 7). Ascorbate is a crucial cosubstrate of enzymes responsible for post-translational hydroxylation of prolyl and lysyl residues, which are necessary for thermostability of the collagen triple helix and for extracellular cross-linking of collagen fibers, respectively. ${ }^{28,29}$ These combined results might reflect an increase in the extracellular matrix within the fibrotic areas.

MALDI-FT-ICR-MSI is a powerful tool that enables spatially resolved and label-free detection of hundreds to thousands of molecules within a single tissue section. ${ }^{10,11} \mathrm{~A}$ broad range of analytes can be investigated, including drugs. ${ }^{10,11,30}$ Thus, it is possible to analyze the distribution and concentration of drugs within a tissue section..$^{30,31}$ Thus, it could be possible to directly compare diseased human tissues treated with chemotherapeutics or other drugs with their correspondingly treated mouse models to analyze drug distribution, behavior, and drug-mediated molecular changes. Such studies could facilitate clinical trials and drug 
development. Our novel protocol is generally suitable for all fields of medical research that compare human tissues with comparable animal model tissues, including research in cancer, neuroscience, and cardiology.

Our novel approach also contributes to the internationally recognized call for replacement, reduction, and refinement (3Rs) of animals used for experimental research. ${ }^{32}$ Just as the protocol can utilize archived FFPE-fixed human samples, it also can utilize FFPE-fixed animal tissues, and thereby spare the lives of additional animals for the validation experiments. The results of the validation experiments may reduce the number of killed animals by identifying animal models that are only weakly comparable with the human disease. The validation experiments also could provide insight on how the animal model could be improved for greater specificity with the disease phenotype. The validation experiments also provide more specific use of the animals for specific research questions (fit-for-purpose validation ${ }^{1}$ ), and reduce the numbers of different animal models used for one study when only one model has the best fit. The high-throughput screening of the animal tissue microarrays can be used to identify inter-animal variability, which also could be used to improve the model.

This study presented a proof-of-concept experiment, and further investigations can improve the experimental outcomes and results. A more sophisticated analysis of the annotated molecules by validation and identification with additional mass spectrometric techniques is possible. It also is possible to integrate both human and mouse material into one TMA for sample randomization. This was not needed in the present experiment because all tissue cores were measured in the same MALDI imaging run. Our purpose was not to identify deep biological results; rather, we developed the new protocol for direct comparisons of human and mouse tissues in a manner that was highly reliable and easy to perform.

In summary, our proof-of-concept study establishes a novel approach that can be used to define metabolomic characteristics of human diseases and translate them directly into animal models. The protocol can be used to validate established or newly engineered animal models, and thereby generate a deeper understanding of the molecular features of a human disease that can be modeled by an induced animal. Clearly, new experimental approaches and novel technological synergisms are required for real progress in the translation of research from bench to bedside.

\section{ACKNOWLEDGMENTS}

A.W. was funded by the Ministry of Education and Research of the Federal Republic of Germany (BMBF; Grant Nos. 01ZX1310B, 01KT16015), the Deutsche Forschungsgemeinschaft (Grant Nos. SFB 824 TP Z02 / C4, CRC/TRR 205 S01) and the Deutsche Krebshilfe (No. 70112617). We thank Ulrike Buchholz, Claudia-Mareike Pflüger, Gabriele Mettenleiter, and Andreas Voss for technical assistance.

\section{DISCLOSURE/CONFLICT OF INTEREST}

The authors declare no conflict of interest.
1. Denayer T, Stöhr TMVR. Animal Models in translational medicine: Validation and prediction. N Horiz Transl Med 2014;2:5-11.

2. Burkhardt AM, Zlotnik A. Translating translational research: mouse models of human disease. Cell Mol Immunol 2013;10:373-374.

3. Seok J, Warren HS, Cuenca AG, et al. Genomic responses in mouse models poorly mimic human inflammatory diseases. Proc Natl Acad Sci USA 2013;110:3507-3512.

4. Van Dyke T. Finding the tumor copycat: approximating a human cancer. Nat Med 2010;16:976-977.

5. Aichler $M$, Seiler $C$, Tost $M$, et al. Origin of pancreatic ductal adenocarcinoma from atypical flat lesions: a comparative study in transgenic mice and human tissues. J Pathol 2012;226:723-734.

6. Hruban RH, Adsay NV, Albores-Saavedra J, et al. Pathology of genetically engineered mouse models of pancreatic exocrine cancer: consensus report and recommendations. Cancer Res 2006;66: 95-106.

7. Olive KP, Jacobetz MA, Davidson CJ, et al. Inhibition of Hedgehog signaling enhances delivery of chemotherapy in a mouse model of pancreatic cancer. Science 2009;324:1457-1461.

8. Emmert-Buck MR, Bonner RF, Smith PD, et al. Laser capture microdissection. Science 1996;274:998-1001.

9. Espina V, Heiby M, Pierobon M, et al. Laser capture microdissection technology. Expert Rev Mol Diagn 2007;7:647-657.

10. Aichler $M$, Walch $A$. MALDI Imaging mass spectrometry: current frontiers and perspectives in pathology research and practice. Lab Invest 2015;95:422-431.

11. Norris JL, Caprioli RM. Analysis of tissue specimens by matrix-assisted laser desorption/ionization imaging mass spectrometry in biological and clinical research. Chem Rev 2013;113:2309-2342.

12. Balluff B, Frese CK, Maier SK, et al. De novo discovery of phenotypic intratumour heterogeneity using imaging mass spectrometry. J Pathol 2015;235:3-13.

13. Alexandrov T, Chernyavsky I, Becker $M$, et al. Analysis and interpretation of imaging mass spectrometry data by clustering mass-to-charge images according to their spatial similarity. Anal Chem 2013;85:11189-11195.

14. Buck A, Ly A, Balluff $B$, et al. High-resolution MALDI-FT-ICR MS imaging for the analysis of metabolites from formalin-fixed, paraffin-embedded clinical tissue samples. J Pathol 2015;237:123-132.

15. Ly A, Buck A, Balluff $B$, et al. High-mass-resolution MALDI mass spectrometry imaging of metabolites from formalin-fixed paraffinembedded tissue. Nat Protoc 2016;11:1428-1443.

16. McDonnell LA, van Remoortere $A$, de Velde $N$, et al. Imaging mass spectrometry data reduction: automated feature identification and extraction. J Am Soc Mass Spectrom 2010;21:1969-1978.

17. Mantini D, Petrucci F, Pieragostino D, et al. LIMPIC: a computational method for the separation of protein MALDI-TOF-MS signals from noise. BMC Bioinformatics 2007;8:101.

18. Mouratis MA, Aidinis V. Modeling pulmonary fibrosis with bleomycin. Curr Opin Pulm Med 2011;17:355-361.

19. Bensadoun ES, Burke AK, Hogg JC, et al. Proteoglycan deposition in pulmonary fibrosis. Am J Respir Crit Care Med 1996;154(6 Pt 1): 1819-1828.

20. Wilson MS, Wynn TA. Pulmonary fibrosis: pathogenesis, etiology and regulation. Mucosal Immunol 2009;2:103-121.

21. Venkatesan N, Ouzzine $M$, Kolb $M$, et al. Increased deposition of chondroitin/dermatan sulfate glycosaminoglycan and upregulation of beta1,3-glucuronosyltransferase I in pulmonary fibrosis. Am J Physiol Lung Cell Mol Physiol 2011;300:L191-L203.

22. Handel TM, Johnson Z, Crown SE, et al. Regulation of protein function by glycosaminoglycans-as exemplified by chemokines. Annu Rev Biochem 2005;74:385-410.

23. lozzo RV. Matrix proteoglycans: from molecular design to cellular function. Annu Rev Biochem 1998;67:609-652.

24. Mizuguchi S, Uyama T, Kitagawa $\mathrm{H}$, et al. Chondroitin proteoglycans are involved in cell division of Caenorhabditis elegans. Nature 2003;423:443-448.

25. Souza-Fernandes AB, Pelosi P, Rocco PR. Bench-to-bedside review: the role of glycosaminoglycans in respiratory disease. Crit Care 2006;10: 237.

26. Kunzke T, Balluff $B$, Feuchtinger $A$, et al. Native glycan fragments detected by MALDI-FT-ICR mass spectrometry imaging impact gastric cancer biology and patient outcome. Oncotarget 2017;8:68012-68025. 
27. Vitiazeva V, Kattla JJ, Flowers SA, et al. The O-linked glycome and blood group antigens $A B O$ on mucin-type glycoproteins in mucinous and serous epithelial ovarian tumors. PLoS ONE 2015;10:e0130197.

28. Hata R, Senoo H. L-ascorbic acid 2-phosphate stimulates collagen accumulation, cell proliferation, and formation of a three-dimensional tissuelike substance by skin fibroblasts. J Cell Physiol 1989;138:8-16.

29. Murad S, Tajima S, Johnson GR, et al. Collagen synthesis in cultured human skin fibroblasts: effect of ascorbic acid and its analogs. J Invest Dermatol 1983;81:158-162.
30. Castellino $\mathrm{S}$, Groseclose MR, Wagner D. MALDI imaging mass spectrometry: bridging biology and chemistry in drug development. Bioanalysis 2011;3:2427-2441.

31. Buck A, Halbritter $S$, Spath $C$, et al. Distribution and quantification of irinotecan and its active metabolite $\mathrm{SN}-38$ in colon cancer murine model systems using MALDI MSI. Anal Bioanal Chem 2015;407: 2107-2116.

32. Russell WMS, Burch RL. The Principles of Humane Experimental Technique. Methuen and Co Ltd: London, UK, 1959. 